\title{
Implementing Authentic Activities for supporting learning through remote monitoring of earthquakes
}

\author{
Nelson Baloian, Stephan Buschmann, Henning Breuer and Mitsuji Matsumoto \\ DCC, Universidad de Chile, Blanco Encalada 2120, Santiago, Chile \\ GITS, Waseda University, 1011 Okuboyama, Nishi-Tomida, \\ Honjo-Shi, Saitama 367-0035, Japan \\ nbaloian@dcc.uchile.cl
}

\begin{abstract}
The discovery or re-construction of scientific explanations and understanding based on experience is a complex process, for which school learning often uses shortcuts. Based on the example of analyzing real seismic measurements, we propose a computerfacilitated collaborative learning scenario which meets many of the requirements for authentic learning. The implementation of the learning environment is based on a general platform for supporting collaborative modeling.
\end{abstract}

\section{Introduction}

Vygotsky's [10] sociocultural theory promotes the importance of social interaction and the use of artifacts for knowledge acquisition. Bellamy [1] proposes three principles for the design of educational environments derived from Vygotsky's works. First, the notion of authentic activities proposes the modeling of activities and tools derived from professional practices. Second, "construction" refers to learners creating and sharing artifacts within their community. Third, educational environments should be designed to involve a close collaboration between learners and their peers as well as between students and experts.

However, activity-theoretic approaches [2] usually remain rather general in relation to implications for the design of tasks and artifacts. In particular, the notion of authenticity widely spread in the current literature on e-learning remains a blurry demand rather than a welldefined or measurable concept.

Reviewing theory and research on authentic activities and online learning, Reeves et al. [9] propose ten characteristics of authentic activities. Authentic activities are supposed to have real world relevance and create valuable products. The learning process is integrated with assessment. It involves ill-defined, complex tasks to be examined from various perspectives, using a variety of resources and allow a diversity of outcomes. They provide opportunities for collaboration and reflection of students' values and can be applied across several subject areas. Instead of a final and universal definition, their additive list of features alludes to the need to define what is meant by authenticity and which requirements for learning can be derived from each definition. In the end, every experience could be considered authentic. However, "authentic" activities should only be those expanding original boundaries of knowledge on a previously specified subject. Following activity theory in starting from a problem-space motivating activities, we specify/ substitute authenticity by real-world problems, tasks and collaboration. Instead of claiming vague authenticity, we propose to design learning environments for the accomplishment of goals and tasks, derived from real-world problems requiring collaboration.

Learning goals and tasks based on real-world problems can be taken from experts' activity structure and their professional tools. According to Vygotsky, experts should provide scaffolding support to learners proximal development whereas students are motivated to participate in communities of .

Regarding collaboration, it is important that the need for collaboration be not artificially imposed on the community of learners by the system but grounded on the task. Learners will appreciate the value of and seriously engage in collaborative activities only if collaboration is really needed to accomplish the task. Thus, it is also important to distinguish between a task to be accomplished alone and one requiring or profiting from collaboration with peers and experts. Understanding and appreciating the need for collaboration may be a meaningful part of the learning process.. 


\section{Distributed seismography}

A valuable application domain to meet these pedagogical requirements is work on distributed seismography. The real-world problem starts with the natural phenomenon of earthquakes experienced by most Chilean students. This country is highly seismic and thus, its inhabitants are good subjects for our research.

Experts use results from seismographic research to analyze seismic processes, to evaluate and avoid regional risks and trying to understand the uncontrollable behavior of nature. Remote measurement and analysis require not only professional tools and practices but also applying mathematics and physics. Collaborative effort is needed to integrate temporal-spatial measures into shared computations and the creation of seismic maps. Besides, persisting complexities and fuzziness in measurement instruments as well as disputes on theoretic approaches afford participants to specify and argue about their sometimes conflicting research decisions and conclusions.

Students may also imitate the experts' work, subject to their incipient knowledge. Thus, we developed an environment consisting of a seismograph network, a computer network that allows the sharing of the data generated and, most important, the tools enabling students and teacher to process this information. This environment lets students learn about geophysics by engaging in seismographic research, methods and tools. They also develop and apply basic concepts and methods of mathematics and physics, discover the potentials of collaboration and reflect upon the impact of scientific research. The following design principles were applied to support these learning goals and provide a computation-augmented environment:

- Orientation on expert workflow, activity structures and tools.

- Visualization supports concept understanding and (re-)creation of common grounds. From a learner's point of view it also provides a means for active, open-ended exploration of scientific questions and demonstration of research results, a basis for collaborative exchange and discussion.

- Integration of online and offline, individual and collaborative, in-class and distributed activities.

- Flexibility to adapt the environment to the local conditions (students' background and capabilities and/or teacher's preferred teaching style)..

\section{Related Work}

The The CoVis project [4] focuses on "learning through Collaborative Visualization" of science implementing authentic practices. It provides a variety of collaboration and communication tools and tries to embed the use of technology in the development of new curricula and pedagogical approaches. It encompasses a project-enhanced science learning pedagogy, scientific visualization tools for open ended inquiry and networked environments for communication and collaboration [4].

Bellamy [1] introduces two systems, which are based on authentic activity theories. The first one, Dinosaur Canyon, designed for teaching earth sciences to middle school students, is a simulation of a canyon, petrology and a paleontology lab. It provides a simulated context for students to engage in the activities of interpretation of rock and fossils. Students work in small groups, each group studying a portion of geological sequence through the canyon. They select a 10 -meter by $10-$ meter square in their area and proceed to collect fossils and rocks and analyze them in the lab. The second one, Media Fusion, allows students to construct digital video messages that can contain embedded pointers to data analysis application. It focuses on allowing students to explore issues concerning global warming. It contains video and text messages created by experts on global warming and global warming data students can explore.

A synthesis between discovery learning in science and collaborative learning, both supported by computational tools has recently been suggested in [6]. Indeed, there is a variety of different collaborative activities in discovery learning and collaborative modeling. Bollen [3] identifies the following aspects of computer support in collaborative modeling:

- Several students can share a running model by synchronizing their simulation environments.

- The actual model building process can be shared by using a modeling language and annotations in shared workspaces.

- Simulations are analyzed to generate hypotheses about the global behavior of systems.

- Data can be collected in a distributed way with several parameters. Shared workspaces enable students to gather data from various groups. 
- Group work can be supervised by a distant tutor sharing the environment.

The "Cool Modes" [7] eases these activities by providing a uniform shared workspace environment which allows constructing and running models with several formal representations (Petri nets, System Dynamics, mathematical graphs, etc.). It also supports semi-formal argumentation graphs and hand-written annotations. The work reported in this paper has been strongly inspired by this development. It uses a variant of Cool Modes as a technical platform.

\section{Learning in a seismic environment}

In Santiago, Chile, six seismographic sensors were installed in various high schools and attached to computers (Fig. 1, dark triangles). There is also a sensor network installed for scientific research in the region (gray triangles).

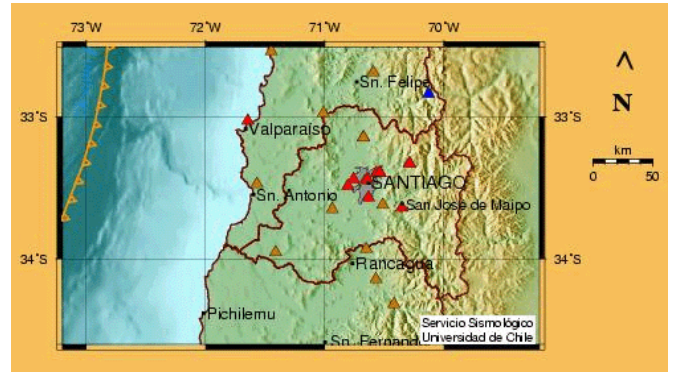

Figure 1. The network of seismic sensors

A group of students is responsible for maintaining and taking care of the sensor and the computer at each school. Whenever an earthquake occurs, the sensors produce data about the intensity of the earthquake. This data is sent to the computer and stored in files. An important goal for students is to calculate the earthquake epicenter. By applying knowledge about how waves propagate in the ground and geometry, every group is able to compute the distance at which the epicenter was located. At least the data generated by two additional (3 in total) sensors with a different location are needed to find the location of the hypocenter and then the epicenter with some degree of accuracy.

The data of these two additional sensors will make possible the localization of the hypocenter of the earthquake by triangulation, so it is important that at least three groups share they data and engage in a collaborative process. They must find the point where all the semi spheres defined by finding the point where all semispheres defined by the distance from the sensors to the hypocenter intersect. The epicenter is the projection of the hypocenter on the ground surface and marks the point where the earthquake had its largest intensity as captured by human beings. Figure 2 depicts this process in a $2 \mathrm{D}$ view. Setting the network of sensors and the servers amounts to just half of the work.

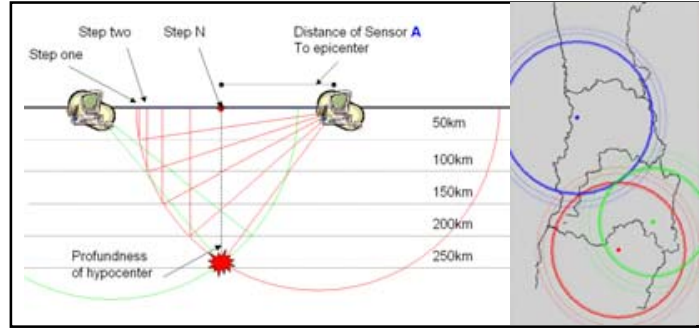

Figure 2: The iterative search for the epicenter

The students must also have a framework where they can do the necessary computations to determine the distance to the epicenter. They should also be able to share the data with all other groups also hit by the earthquake. Finally, they must be able to share and discuss the results with the remote groups in order to learn collaboratively. The need to collaborate follows from the procedure to find the epicenter, as explained in Section 5. The students use a program implementing the following functionalities:

- Retrieve data from the local seismograph and publish them on a server

- Download data from remotely located seismographs

- Provide a framework where students can do their computations and graphic operations in order to find the epicenter

- Provide a framework to compare the results obtained by other groups

The most interesting feature of the system is the way it supports the students in their calculations and graphics. It provides a working area for this purpose, which is meant to support the workflow of the students' activities. A workflow is represented as a network of different types of nodes, each one implementing a further step towards the calculation of the epicenter. The nodes have different functionalities and appearance (Fig. 3). Nodes can be created and placed in the working area by "drag-and-drop" from a palette of different node types. Adding an edge between two nodes transfers output values of one node as input values for the successor, but of course, this is allowed by the system only between nodes where this operation makes sense. 


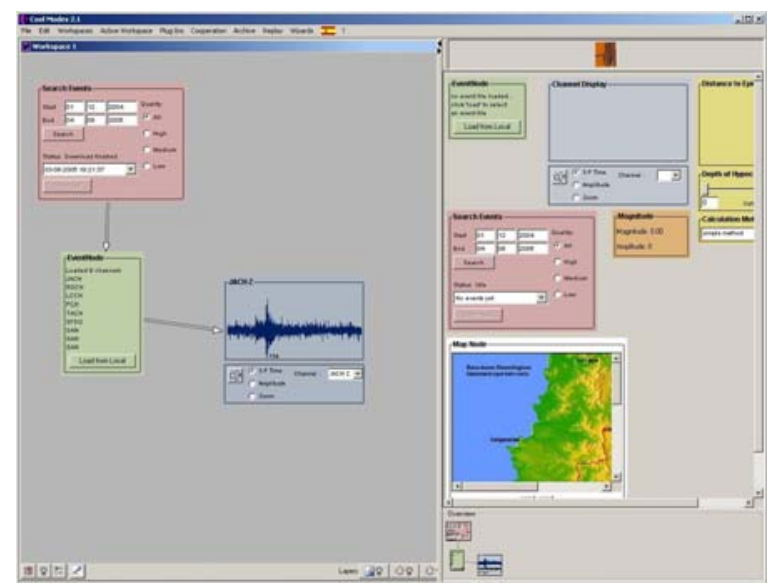

Figure 3. The workspace with different kinds of nodes

One type of node (the first in the sequence of Fig. 3) is able to search for events in the repositories. Another (second in the sequence) can read and store the data of a file generated by a seismic sensor. It also displays some useful information like date and duration of the event. Another type of node (the third of Fig. 3) is able to graphically display this information, if the students connect them with an arrow. Thus, the students can easily determine the time lag between the primary and secondary wave, just by marking this space in the graphic node (as seen on Fig. 1). The student can also zoom in and out, scroll or mark relevant data points. The determined time lag is the basis for further calculations as mentioned above. The "calculation node" uses this value to compute the distance dependent on the time lag and the iteratively chosen depth. Establishing a connection with another node called "Map Node" displays the map of the specific region e.g., Santiago, and the computed distances as well. Using this two-dimensional top view the minimum of the intersection can easily be found. In this way, this nodes network offers a workflow to exchange results and/or intermediate data.

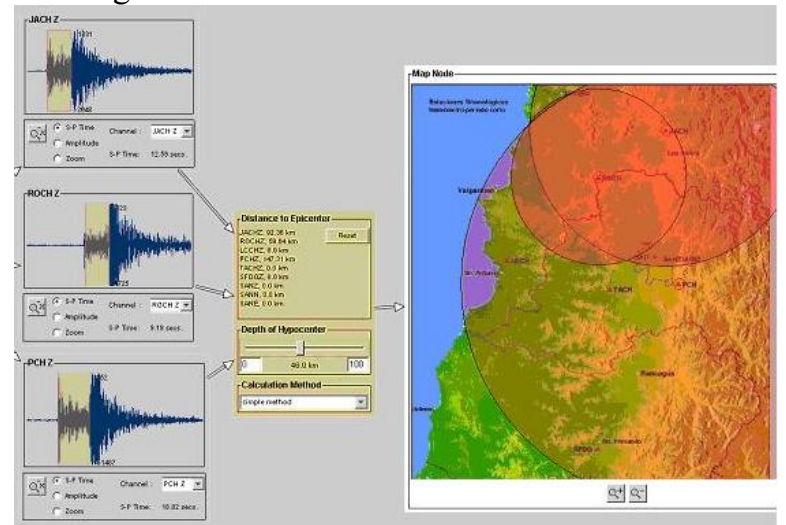

Figure 4. searching for the epicenter with data generated by three sensors
The system was implemented on top of CoolModes with the specific aim of binding the Concept Mapping tools with archiving and retrieving functions. Thus, the results of a cooperative knowledge can be stored and retrieved [5].

\section{Learning by Collaborating}

According to Bellamy [1], three principles for the design of educational environments have been derived from Vygotsky's work:

- Authentic activities: Children should have access to, and participate in, similar cultural activities to those of adults and should be using ageappropriate tools and artifacts modeled on those used by adults. The system creates the environment for authentic activities because it gives the possibility for the students to mimic the activities professional people do while monitoring and recording earthquakes, as well as calculating some characteristics of them. The system gives the appropriate scaffolding for doing transformation of data and calculating complicate formulas.

- Construction: Children should be constructing artifacts and sharing them with their community. Freestyler documents [5] enable the collaborative construction of the workflow for calculating the characteristics of the earthquake, which they can share, with the rest of the community. In the next chapter we will see how students can construct physical artifacts to model the earthquake.

- Collaboration: Educational environments should involve collaboration between experts and students and between individual learners and fellow learners.

This setting allows different kinds of collaborative learning activities:

Collaboration inside one group: the group trying to compute the distance to the hypocenter, based on local data. The tool supports asynchronous collaboration by annotating and recording the work of each participant. Creating coupled sessions supports synchronous distributed collaboration. For this the tool was integrated with MatchMaker [ref].

Collaboration among groups in the same earthquake region: exchanging data produced by the seismograph is the first step towards collaboration. 
Calculation of the distance from a seismograph to the hypocenter is based on visually determining the time difference between the arrival of both waves. Since calculating the distance to the hypocenter is based on a visual procedure. This will necessarily mean, the results of the different groups will not be exactly the same. The system gives the necessary platform for the groups to engage in a discussion, trying to find the most probable area where the hypocenter was located, contrasting all the results.

Collaboration among groups in different regions: because the system is working over the Internet, it gives student groups located in remote areas the possibility to use the same data, ask about the consequences of the earthquake and try to "reproduce" it in the virtual laboratory.

\section{Conclusions}

The system has been successfully used for already about one year in three schools with students of the $11^{\text {th }}$ school year. The way this tool fits in the general curricula is by using it to teach geology and physics. The idea of installing sensors in schools is not new: there are some similar initiatives in USA, France and Japan. The novel idea is to use them for collaboration at various levels. The main contribution of this work is to present a platform supporting cooperative learning in schools with very little economical resources. In fact, two of the three schools have just the sensor (which was donated by the Universidad de Chile) and 2 or 3 computers connected to the internet. The third one corresponds to a private school and has more resources. However, results in the other two schools are also very encouraging and it is being used as a teaching instrument regularly. Although they faced all the typical problems of a low budget project, the experience was very enriching for students and teachers (a more formal analysis of the results is out of the scope of this paper).

This work is part of a larger project named Coldex (http://www.coldex.info), which deals with the problem of achieving true learning through remote collaborative monitoring or experimentation. In our opinion, this is only the first part of the work, which should be done in order to achieve meaningful learning through remote or distributed collaborative experimentation. There must also be a system supporting the learning process through concrete learning activities.

The work described in this paper allows various types of collaborative learning, since the results of others are needed for own work, and vice-versa. The collaborative opportunities provided by the setting occur naturally. This is perhaps the main difference with other collaborative learning experiments in which the collaboration is artificially induced.

\section{References}

[1] Bellamy, R.K.E. (1996). Designing Educational Technology: Computer-mediated Change. In Nardi, B. A. (Ed.). Context and Consciousness: Activity Theory and Human-Computer-Interaction (pp. 123-146). Cambridge, Massachusetts: MIT Press.

[2] Bertelsen, O.W. \& Bodker, S. (2003). Activity Theory. In Caroll, J.M. (ed.). HCI Models, Theories and Frameworks. Toward a multidisciplinary science (pp. 291-324). New York: Morgan Kaufmann.

[3] Bollen, L., Hoppe, H.U., Milrad, M. \& Pinkwart, N. (2002). Collaborative Modelling in Group Learning Environments. In Davidsen et al. (eds), Proceedings of the XX International Conference of the System Dynamics Society. Palermo (Italy), July 2002.

[4] Edelson, D.C. (1997). Realising Authentic Science Learning through the Adaptation of Scientific Practice. In Tobin, K \& Fraser, B. International Handbook of Science Education. Dordrecht, NL: Kluwer.

[5] Hoppe, H. U. \& Gaßner, K. (2002). Integrating collaborative concept mapping tools with group memory and retrieval functions. Proc. of CSCL-2002. Boulder, Colorado (USA), January 2002.

[6] Joolingen, W. R., van (2000). Designing for Collaborative Learning. In G. Gauthier, C. Frasson \& K. VanLehn (Eds.) Intelligent Tutoring Systems, 5th International Conference, Montréal, Canada. Berlin: Springer.

[7] Pinkwart, N., Hoppe, H. U. \& Gaßner, K. (2001). Integration of Domain-Specific Elements into Visual Language Based Collaborative Environments. In M. R. S. Borges, J. M. Haake \& H. U. Hoppe (Eds.), Proceedings of the 7th International Workshop on Groupware, CRIWG 2001.Darmstadt, Germany, 6-8 September 2001. IEEE CS Press.

[8] Quintana, C., Carra, A., Krajcik, J., \& Soloway, E. (2001). Learner-Centered Design: Reflections and New Directions. In J.M. Carroll (Ed.), Human-Computer Interaction in the New Millennium (pp. 605-624). Reading, Massachusetts: Addison-Wesley.

[9] Reeves, T. Herrington, J., \& Oliver, R. (2002). Authentic Activities and online learning. In J. Herrington (Ed.) Proceedings of HERDSA. Joondalup: Edith Cowan University.

[10] Vygotsky, L. S. (1978). Mind in society: the development of higher psychological processes. Cambridge: Harvard University Press. 